\title{
Study on Giuseppe Castiglione, a Court Painter in the Qing Dynasty
}

\author{
Ran Geng \\ Shanghai Publishing and Printing College, Shanghai, China \\ Email: 928274224@qq.com
}

How to cite this paper: Geng, R. (2021). Study on Giuseppe Castiglione, a Court Painter in the Qing Dynasty. Open Journal of Social Sciences, 9, 193-203.

https://doi.org/10.4236/jss.2021.99014

Received: August 16, 2021

Accepted: September 6, 2021

Published: September 9, 2021

Copyright $\odot 2021$ by author(s) and Scientific Research Publishing Inc. This work is licensed under the Creative Commons Attribution International License (CC BY 4.0).

http://creativecommons.org/licenses/by/4.0/

(c) (i) Open Access

\begin{abstract}
At all times and in all countries, exchanges between China and the west are always a constant topic. From the Qing Dynasty Lang Shining Chinese and Western painting, a new form of painting Chinese painting formed after absorbing some of the characteristics of Western painting. In this paper, through the comparison and research between painting and Chinese traditional meticulous painting subjects Chinese and Western Qing missionaries painter Lang Shining, to explore the characteristics of Lang Shining new body painting and realistic significance to modern fine brushwork of Chinese and Western fusion.
\end{abstract}

\section{Keywords}

Chinese and Western Painting, Lang Shining, Giuseppe Castiglione, Traditional Meticulous Painting, Practical Significance

\section{Introduction}

In Chinese history, the Qing Dynasty was a period of frequent integration of Chinese and Western paintings, which was caused by that many European missionaries came to China at that time and brought in western culture, art and other resources. Some missionaries were appreciated by the emperor at that time and became court painters. Lang Shining was one of them, who was an important part of the communication between China and the west at that time and made great contributions to the development of Chinese painting. The origin of all this began when Giuseppe Castiglione came to China to deliver a sermon.

\section{Giuseppe Castiglione's Life Story and Artistic Achievements}

Castiglione, formerly known as Giuseppe Castiglione, was born in Milan, Italy in 
1688. According to the History of Sino-foreign Art Exchange, he learned painting from Andrea Pozzo at his early age. He joined the Church of Jesus in 1707. Giuseppe Castiglione has been very well trained in painting and commanded a deep foundation of Western painting, all of which can be found in the western paintings he left over. In 1715, he came to China as a missionary. Emperor Kangxi of Qing Dynasty loved western paintings very much. However, Giuseppe Castiglione was very talented and his painting style was exquisite. He was soon summoned by Emperor Kangxi. From then on, he began his painting career in the palace for more than 50 years.

He integrated Chinese and Western painting techniques, including portraits, animals, flowers and birds, landscapes, etc., covering a wide range of areas. $\mathrm{He}$ was an all-round talent in art, who has contributed to the cultural and artistic exchanges between China and Europe at that time, and thus occupied a place in the history of Chinese art. He served successively for the three emperors of Kangxi, Yongzheng, Qianlong in the Qing Dynasty. In his later years, he was presented with Third-Level Official Cap, and after his death, he was presented the title of assistant minister. He was originally a missionary of Society of Jesus in China, but he had been busy painting for the emperor and had no chance for preaching. In order to please the emperors, he was very good at adopting Chinese painting techniques and maintaining the basic characteristics of Western art. Therefore, his works are very distinctive (Su, 1998).

In this long period of more than 50 years, his main contribution is to boldly try the methods used in Western painting and integrate Chinese and Western painting, that is, Giuseppe Castiglione's new style painting, especially the combination with Chinese meticulous painting, has made outstanding achievements. The main characteristics of the new style painting are: solid realistic painting ability, flexibility of lines, picture polishing layer upon layer, and uniqueness and harmony of use of color, which was integrated with the Western painting method, and paid attention to the anatomical structure, three-dimensional, space and light and shadow effects, not only possessing the overall summary of European oil painting realism, but also the pen-and-ink interest of Chinese painting.

He used this painting method to create more than 80 works, involving characters, pommel horse, flowers and birds and landscapes, documentary events, etc., with a wide range of subjects. His portraits of emperors are vivid and expressive, particularly excellent. His representative works include: Qianlong Court Dress Scroll, Portrait of Hongli and His Queens, Emperor Qianlong's Bust, etc. He also presided over and completed many great works of great historical and documentary significance, such as the Painting of Banquet Awarded in Wanshu Garden, Equestrian Painting, etc. China's earliest copper engraving work of the Battle Map of pacifying Huai and Hui Tribes was also completed under his creative leadership .Among above, the works with the theme of exotic flowers and plants are also superb. Famed magnum opus such as the Painting of Bird Offering British Iris, the Painting of Holy Images Gathering, etc. established his position as a court painter. In addition, Giuseppe Castiglione also participated in the 
design of western style buildings in the Summer Palace. He also helped and guided Nian Xiyao-the Huaihe River Supervisor to complete China's first monograph on the perspective principles of Western painting the Study on Viewing (Nie, 1995).

Through the above analysis of Giuseppe Castiglione's artistic achievements, it can be seen that he has made a great breakthrough in the integration of Chinese and Western painting. In addition, Giuseppe Castiglione's Chinese and Western painting skills and expressive ability are quite excellent. In the meantime, he has a profound cultivation of Chinese and Western aesthetic quality, which shows his understanding of different styles of Chinese and Western paintings, especially in the combination of Chinese meticulous painting, playing a certain reference role in modern meticulous painting, and his paintings are very representative.

\section{Comparison between Giuseppe Castiglione's Combination Painting of Chinese and Western Elements and Chinese Traditional Meticulous Painting}

Chinese meticulous painting has a history of more than 2000 years, as an independent painting species. Fine brushwork has shown better adaptability in the process of integration of Chinese and the Western elements, since there are many common characteristics of the two: coloration is mainly based on the technique of polishing layer upon layer, the use of color to pursue the harmony of color, the modeling is required to be rigorous, and the painting objects are required to be realistic at the same time. The common characteristics determine that Chinese meticulous painting will have more adaptability in the process of integration of China and the West elements. These painting characteristics can be reflected in Giuseppe Castiglione's paintings, whether figures, flowers, birds, insects, fish, birds and animals, all of them are depicted in detail.

Since traditional Chinese meticulous painting was still the mainstream of art at that time, Giuseppe Castiglione had no similar experience to learn from before. Therefore, it is not a simple matter of skillfully integrating different factors in Eastern and Western painting and letting the Chinese accept it. Comparing Giuseppe Castiglione's works with the excellent works of Chinese traditional meticulous painting, the author makes the comparison of the two aspects of his portrait painting and flowers and birds painting, and finds out the similarities and differences between the two, and looks for some beneficial factors for the integration of Chinese and Western painting. (Chen, 2007)

\subsection{In the Aspect of Human Portrait}

Due to the lack of anatomical knowledge of Chinese painters, they were limited in modeling and structuring. Therefore, at the beginning of the introduction of Western painting, the most amazing thing was the Western portrait painting. In the history of silent poetry of the Qing Dynasty, Jiang Shaoshu recorded in Silent Poetic History: "Matteo Ricci brought the statue of the God of the western regions, which was a woman holding a baby, and her eyebrows and clothes were 
like the shadow of a mirror, seeming to move expectantly. Her dignity and grace cannot be painted by Chinese painters." The true-life characteristics of Western painting being closest to the visual reality, makes Giuseppe Castiglione a sudden rise in the portrait, and greatly appreciated and recognized by Chinese people.

Compared with other kinds of paintings, Giuseppe Castiglione's portraits of emperors and empresses are undoubtedly the most outstanding works. Among them, the Painting Scroll of Qianlong Court Clothes has reached the highest level of portraits of emperors and empresses in all dynasties. Comparing the Painting Scroll of Qianlong Court Clothes with the Sitting Statue of Jiajing Emperor (Ming Dynasty) (Figure 1) and the painting of Kangxi in Court Dress (early Qing Dynasty) (Figure 2) painted by Giuseppe Castiglione, it is difficult to see the portraits of queens before the Song Dynasty due to the factors of age and preservation. From the perspective of the remaining portraits before the Song, Yuan, Ming Dynasties and Qianlong period, they all used the traditional Chinese drawing method of outline drawing, drawing the outlines of the face and clothing with ink lines, and then colored and flat painted with pastel. The picture is monotonous and unchanged. Generally, the best painters in the court or at that time painted for the emperors. Therefore, it can also reflect the development status and level of portraits of emperors and empresses in the Ming and Qing Dynasties (Nie, 1999).

The composition of the three portraits of the emperors adopts the traditional Chinese upright sitting style. The emperor sits in the middle of the picture to show the dignity of imperial power. The Sitting Statue of Jiajing Emperor in Ming Dynasty can show the characteristics of early emperor portraits. The traditional flat painting method is used to depict evenly, and the picture is symmetrical, rigid and inflexible, which makes the whole work lack of rich changes.

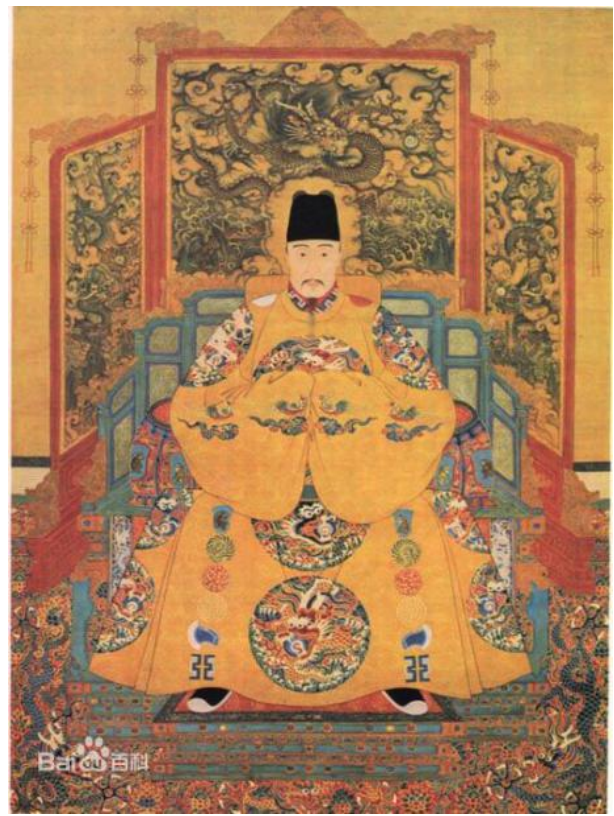

Figure 1. Sitting statue of Jiajing emperor. 


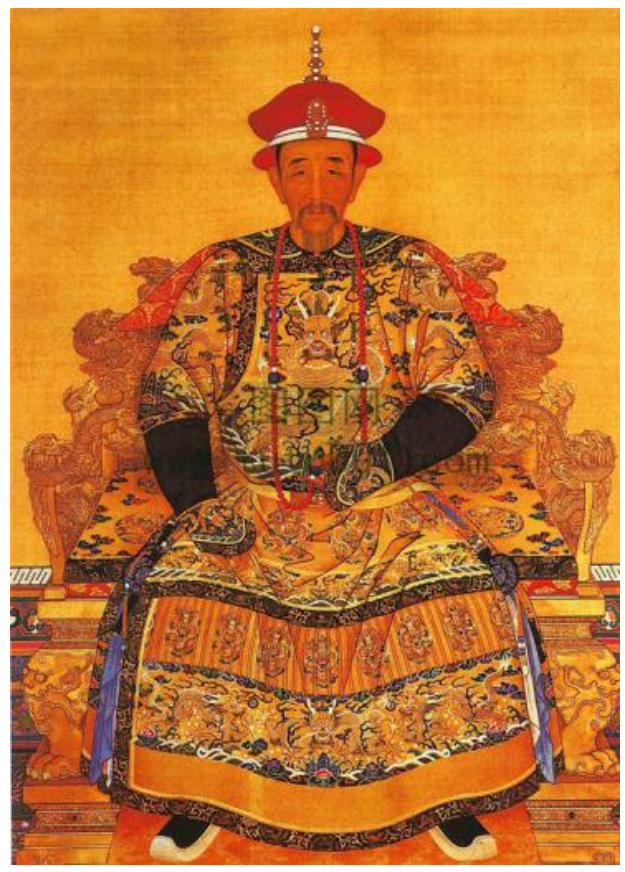

Figure 2. Kangxi in court dress.

During the reign of Emperor Kangxi, there were some western missionaries in the Qing Dynasty. Therefore, Western painting techniques have been used in the portrait of Kangxi in Court Dress (Figure 2). The face of the character presents three-dimensional feeling, but his eyes are still dull and in lack of vivid color expression. Among them, the facial lines of the character are obviously weakened, the eyes and cheekbones have been polished according to the human musculoskeletal structure, and the depiction of hands is more stereoscopic and realistic than the previous Sitting Statue of Jiajing Emperor, but it is not particularly strong. In addition, the modeling is not very rigorous, and the color of the character is dark, leading to the weakening of the overall momentum of the character, and lack of imperial momentum.

The painting of Kangxi in Court Dress (Figure 2) is an immature work in the combination of Chinese and Western painting, which is a transitional work. Giuseppe Castiglione's painting of Painting Scroll of Qianlong Court Clothes (Figure 3) is already a mature work combining Chinese and Western culture. The Emperor Qianlong in the painting just ascended the throne in 1736, and the emperor in the painting is high-spirited and vigorous. His majesty and reign of the world are fairly translated in the painting .Among them, Giuseppe Castiglione's western realistic painting technique makes the five facial features portrayed by the emperor detailed, accurate, three-dimensional and lifelike. In order to meet the aesthetic habits of the Oriental people, the light source of the picture is used as the positive light source, thus avoiding the phenomenon of "the face changing unpredictably" and making the facial features very clear. Lines are rarely used in the technique. Eyebrows are outlined in light of the structure with slightly heavier ink. The corners of the mouth are slightly upturns, and the nose shows a little 


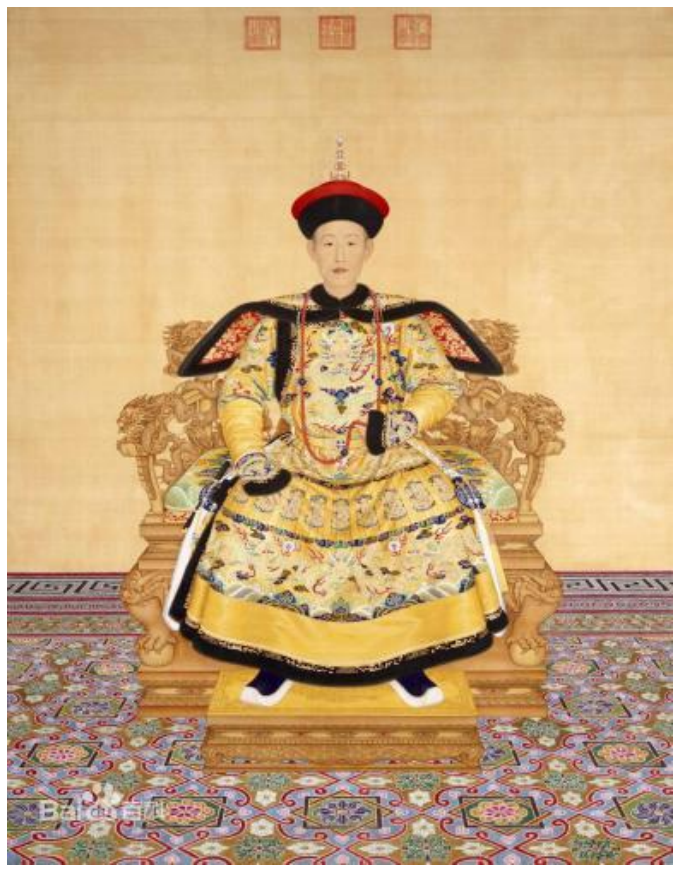

Figure 3. Painting scroll of Qianlong court clothes.

highlights. Meanwhile, the structure and block surface relationship of the five sense organs are well interpreted, and the relationship between muscle and bone is depicted easily and naturally, which makes the characters in the whole work look high spirited and sufficiently shows the emperor's noble, calm, self-confident and natural and unrestrained demeanors. What is worth praising is the depiction of the hand, which is true and delicate with the accurate modeling, so that people can feel the elasticity and warmth of the skin. (Zhang, 2010)

In addition, there are many representative patterns and the texture of clothes are worthy of attention, such as the accessories of characters and the rough edge of collar. It has to be admitted that Giuseppe Castiglione's strong realistic skills are admirable. It's hard to imagine that this is a fine brushwork with nearly 300 years of history. Even in modern times, it is difficult to cross the level of portrait painting. But it is a necessity to take the essence of his painting skills but not copy all copies. Giuseppe Castiglione's realistic and visual effects are worth learning from, but he lacks the spirit of Chinese meticulous painting. In contrast, the Portrait Painting of A Man in Ming Dynasty (Figure 4), which was created by the folk painter, is of typical representativeness. The rich expressive force of Chinese painting lines is incisively and vividly displayed by the author. Eyebrows, eyes and moustaches are outlined with different lines of hardness, softness and strength, and are slightly dyed with faintness. Among them, Li Rihua's "puffy eye" and the skin texture of middle-aged people are extremely interesting, such as Ge Yinliang's "big and small eyes", Luo Yingdou's drooping eyelid due to the old age, He Bin's sunken orbit, etc. The painting is extremely meticulous and full of vivid and flexible beauty. It is a masterpiece of Chinese fine brushwork portrait and figure painting. 


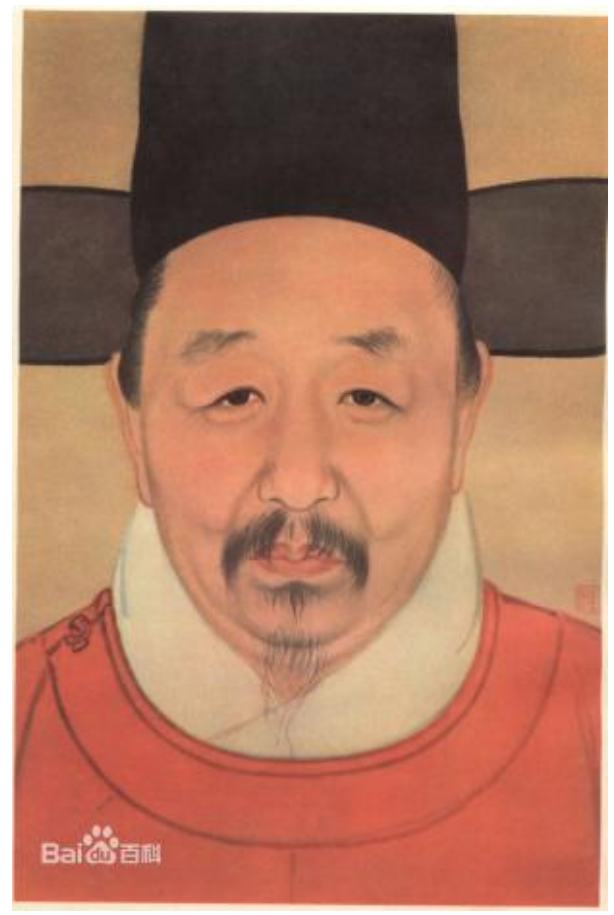

Figure 4. Portrait painting of a man in Ming Dynasty.

\subsection{The Bird-and-Flower Painting}

Compared with portrait painting, Giuseppe Castiglione's attainments in thebird-and-flower painting are slightly inferior. In the $18^{\text {th }}$ century, Western painting was influenced by neoclassicism and the Renaissance. In his early works of the-bird-and-flower painting, his overall communication and color use are similar to those of western still life paintings. For instance, the Painting of Holy Images Gathering is real but looks a little stiff. As a court painter, Giuseppe Castiglione actively received the essence of Chinese bird-and-flower painting, studied the composition form and processing method, improved his painting method while retaining the Western modeling language, formed his own unique painting style, and occupied a place in the Chinese the-bird-and-flower field in a unique form.

If the Painting of Holy Images Gathering is an immature early work, the Painting of Offering British Iris is a more mature representative of bird-and-flower painting. This painting is made for the Longevity Festival of Shizong of Qing Dynasty, which is painted with pines, white eagles, ganoderma lucidum, rocks, flowing water, etc. All of these objects have good connotations of longevity and auspiciousness in Chinese paintings. The western painting forms are used to combine the Painting of Offering Birthday Congratulations (Figure 5) perfectly and realistically, and have always maintained the characteristics of "auspicious prosperity" in court paintings. Giuseppe Castiglione not only absorbed the expression method of Chinese painting, but also understood some national culture of China. White eagle with polishing method, rarely uses line, volume, light and shade, structure, space performance, which is well expressed. The unique texture of each 


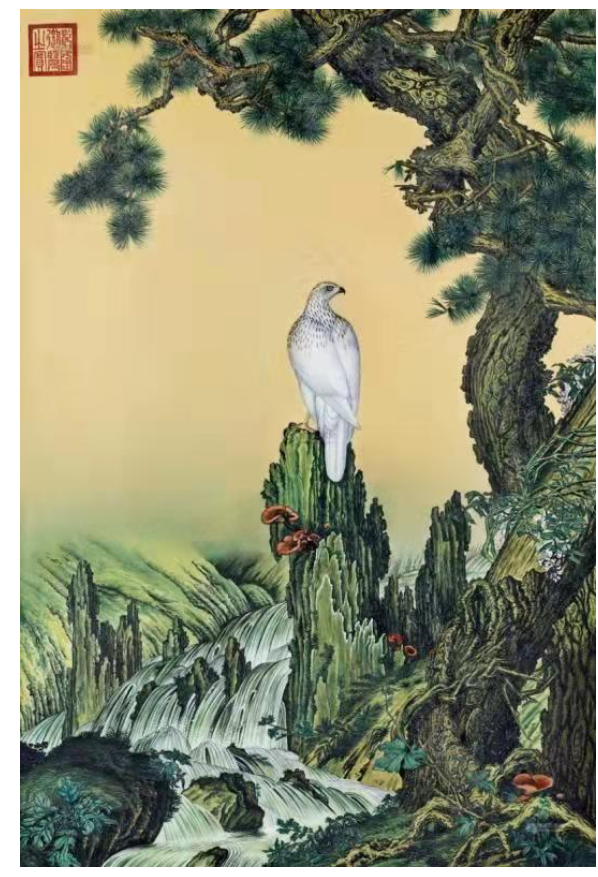

Figure 5. Offering birthday congratulations.

object is concerned. The light sense of flowing water, the texture of white eagle feather and the texture of pine needles are all well differentiated and form a strong contrast. The color of the whole painting is strong and bright, which looks like the description of animal specimens. It is a little stiff and lacks the beauty of flexibility. Similarly, Giuseppe Castiglione's fine brushwork of flowers and birds still scores a success by shape.

On the contrary, the flower-and-bird paintings in the Five Dynasties and Two Song Dynasties present the beauty of both form and spirit. Huang Jian, a famous painter of Western Shu in the Five Dynasties period, served the court as well as Giuseppe Castiglione. By comparing their works, the fundamental differences between them can be found out.

Huang Jian, a painter of Western Shu in the Five Dynasties, was dedicated to the imperial court at the age of 17 . He was especially good at flowers, birds, grass and insects. His painting style was neat and rich, and the color was elegant. The Painting of Rare Birds (Figure 6) is his only remaining work, which was used for his son Huang Jubao to learn painting. There are 24 insects, birds and turtles in total. Using the very fine lines to draw, the line of bird's beak is strong and that of the feather is soft. From the technical form point of view, the painting pays attention to sketching, which is similar in form and real in spirit, with great interestingness. Although there is not too much color rendering, it has a strong aesthetic feeling. Both modeling and realistic painting are not inferior to Giuseppe Castiglione, not only limited to that. His whole picture presents a kind of interesting and wonderful aesthetic feeling, which can be said to be better than Giuseppe Castiglione's. The color is beautiful and elegant, gorgeous but not vulgar, and has the image of royal wealth. 


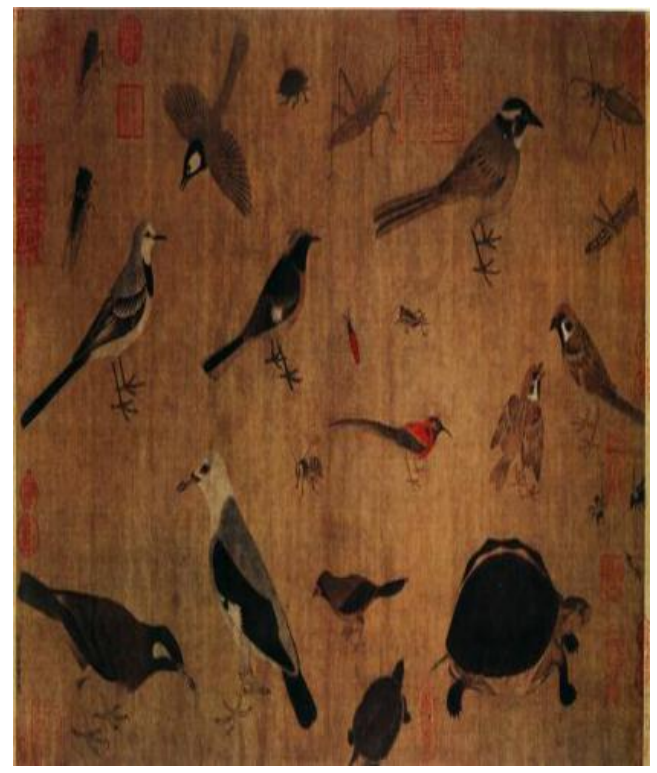

Figure 6. Painting of rare birds.

Cui Bai, a painter of Imperial Painting Academy in the middle of Northern Song Dynasty, is good at painting flowers and birds, especially good at painting from nature. His paintings of geese, cicadas and birds can be called "three wonders". The Painting of Double Happiness (Figure 7) is a masterpiece handed down from generation to generation. In the painting, the dead branches are broken down, the leaves are falling, and the grass is lying on the ground, turning to be a rustle in the air. Two birds with brilliant expressions, give people the feeling of being in the scene. The combination of painting and writing is embodied in drawing, and the brushwork is sophisticated. (Yu, 2007)

From the perspective of visual effect, Giuseppe Castiglione's paintings are fresh and exciting with strong sense of volume, while Cui Bai's works, as a whole, seek subtle changes between objects and images in the plane, and express a kind of dynamic beauty implicitly and peacefully. His superb drawing from nature and meticulous observation has to be marveled. It can be seen that it is a wonderful work with a blend of love and scenery. It can be also seen that drawing from nature of the Song Dynasty was not confined to the simple description of form and seeking truth, but creating a specific artistic conception through the subtle depiction of the form. Therefore, although Giuseppe Castiglione's flower-and-bird paintings are created with more western production techniques, they still lack the charm of beauty compared with the flower-and-bird paintings in the Five Dynasties and Two Song dynasties.

Although fine brushwork figures and fine brushwork flowers and birds are coordinated for meticulous painting, there are still differences between them. As early as the Five Dynasties and Two Song Dynasties, meticulous flower-and-bird painting have been very mature. Among them, Huang Jian, Xu Xi, Cui Bai and other painting masters have created many dazzling treasures for China's meticulous flower-and-bird painting. The form, spirit and meaning have achieved high 


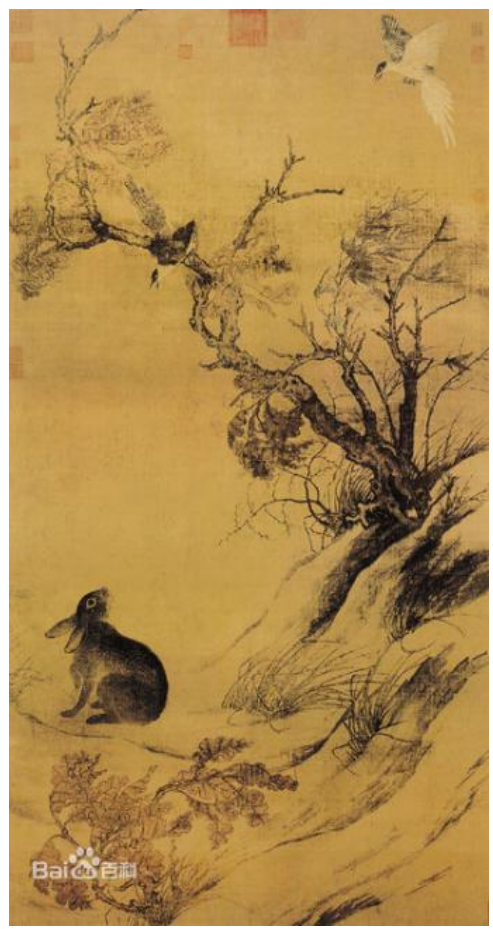

Figure 7. Painting of double happiness.

attainments in the history of Chinese art. Giuseppe Castiglione's flower-and-bird paintings, which are mainly based on realistic techniques, have little advantage in China. The ancient Chinese fine brushwork flower-and-bird painting is no inferior to Western classical painting in realism and artistry, and the artistic concepts pursued by the two are different. The Western pays attention to the rational and scientific research and description of still life, pursuing the expression of light and shadow, space, volume, etc., while Chinese flower-and-bird painters pay more attention to the depiction and expression of plants and birds of real life, so as to express their subjective feelings, place their beautiful thoughts or pursuit of beautiful things, and connect the images with emotion, morality and selfcultivation, thus the effect of artistic conception of blending the emotion into the scene can be attained. From this point of view, Giuseppe Castiglione's Western classical techniques in realistic techniques and composition color, and finally to the art research do not have much room for breakthrough and development, thus which does not have a great impact on Chinese traditional meticulous flowerand-bird painting, and can be inevitable.

\section{New Life of Arts Born in Disputes}

In fact, Giuseppe Castiglione's paintings have always been controversial. As a Westerner, Giuseppe Castiglione's aesthetic and previous art research are based on the Western painting as the main part and the Chinese painting as the supplement. As a person who is learning Western painting, Giuseppe Castiglione's complex emotions can be felt by the author while exploring and understanding Western painting. Giuseppe Castiglione lived in a time when the East and the 
West did not know each other very well. Art exchanges and attempts were very difficult for the society at that time. He was in the field never explored with no experience and precedent. It is really difficult for him to create by relying on his talent, his own cognition of paintings and comprehensive understanding of culture, which is worth learning from. His painting skills are superb and proficient, and the paintings he has been exposed to in China for more than 50 years are the finest and excellent works. The reason why his works are controversial is the lack of Chinese traditional culture, or the difficulty of accepting the new style of painting which is "neither Western nor Chinese" (Kong \& Zhang, 2002).

Giuseppe Castiglione's new style of painting has not been developed in China. In fact, after Giuseppe Castiglione's death, the Western painters in the court were not very accomplished with no strong follow-up powers. Therefore, this new style of Chinese and Western painting began to decline. In the final analysis, the maintenance of this phenomenon was only a preference from Emperor Kangxi. Therefore, the road of integration of China and the West in that era was very harsh.

Nowadays, in this gradually westernized social environment, a teaching system with Western realism as the main content has been formed. The long-cherished wish of pursuing the three-dimensional and realistic feeling of pictures has become more and more straightforward. The blind pursuit of Western art and the pursuit of the so-called modern sense have become everywhere. On the contrary, Western art has never sacrificed its own painting characteristics to blindly follow foreign culture when facing Chinese art. After all, there are great differences between the Eastern and Western cultures. When taking in foreign cultures, there is always a necessity for us to keep a clear mind and start from spiritual ballast and aesthetic pursuit to inherit and carry forward our traditional things, so as to make modern meticulous painting and its spirit reach a new level and height, realize the value of national art, and inject new blood into the world art.

\section{Conflicts of Interest}

The author declares no conflicts of interest regarding the publication of this paper.

\section{References}

Chen, S. N. (2007). A Comparative Study on the Concept of Form and Spirit in Chinese and Western Paintings. Oriental Publishing House.

Kong, X. M., \& Zhang, P. (2002). Comparison of Chinese and Western Arts. Shandong Pictorial Press.

Nie, C. Z. (1995). Foreign Painters in the Qing Dynasty and the Change of Palace Painting Style. Art Research, Issue 1.

Nie, C. Z. (1999). Court Painting in the Qing Dynasty. Shanghai Science and Technology Press.

Su, L. Q. (1998). Biography of Giuseppe Castiglione. China Literature Press.

Yu, J. H. (2007). Xuanhe Painting. Jiangsu Fine Arts Publishing House.

Zhang, G. H. (2010). A Study of Giuseppe Castiglione's Figure Paintings during the Reign of Emperor Qiaolong. Zhejiang University of Technology. 